\title{
A semântica de vetores: uma proposta de análise para os adjuntos temporais
}

\section{Vector space semantics: a proposal for temporal adjuncts}

\author{
Renato Miguel Basso* \\ João Francisco Bergamini-Perez ${ }^{* *}$
}

\section{Resumo}

Neste artigo, depois de revisarmos algumas propostas de análises para os adjuntos temporais 'em x tempo' e 'por x tempo', apresentaremos uma nova análise para esses e outros adjuntos temporais, como 'durante x tempo', 'de x à y tempo' e 'até x tempo', com base na teoria da "semântica de vetores". Essa teoria, primeiramente desenvolvida para lidar com preposições, pode ser expandida para analisar outros tipos de construções, como aquelas que envolvem predicados graduais. Neste texto, analisaremos com a semântica vetores a combinações de adjuntos temporais com o aspecto perfectivo e diferentes tipos de sintagmas de medida (SMs) que podem acompanhar tais adjuntos.

Palavras-Chave: adjuntos temporais; eventos; acionalidade; aspecto verbal; semântica formal.

\section{Abstract}

In this paper, after reviewing some analysis for the temporal adjuncts 'em $\mathrm{x}$ time' and 'por $\mathrm{x}$ time', we present a new analysis for these and other temporal adjuncts, such as 'durante $\mathrm{x}$ time', 'de $\mathrm{x}$ a $\mathrm{y}$ tempo' and 'até $\mathrm{x}$ time', based on a theory known as 'vector space semantics'. This theory was first proposed to deal with prepositions, and was then expanded to deal with other types of constructions, such as those involving gradual predicates. In this paper, we analyze the combinations of temporal adjuncts with the perfective aspect and different types of measure phrases that can accompany such adjuncts using the tools of vector semantics.

Keywords: temporal adjuncts, events, actionality, verbal aspect, formal semantics.

\footnotetext{
* UFSCar/CNPq.

** UFSCar. Agradecemos o apio da FAPESP, processo 2014/10894-1.
} 


\section{Introdução}

s adjuntos temporais são uma das principais ferramentas para classificar e analisar os diversos tipos de eventos e suas interpretações nas línguas naturais. Esse tipo de adjunto é exemplificado por construções como 'por X tempo' e 'em X tempo', em que 'X tempo' está por uma medida de tempo qualquer, e serve, por exemplo, para identificar eventos (a)télicos e durativos.

O recurso a esses adjuntos é, de fato, um marco nos trabalhos em semântica de eventos e pode ser notado desde os trabalhos mais antigos e clássicos sobre eventos e sua natureza (cf. VENDLER, 1957; KENNY, 1963; DOWTY, 1979), até trabalhos mais recentes. Contudo, seu uso limita-se, muitas vezes, a certas combinações específicas - 'em X tempo' com eventos télicos perfectivos e 'por $\mathrm{X}$ tempo' com eventos atélicos imperfectivos -, enquanto que há, na verdade, diversas outras combinações possíveis cuja investigação pode levar a um melhor entendimento não apenas dos adjuntos e seu papel, mas também das distinções entre eventos ${ }^{1}$.

Além disso, há outras duas questões que merecem, a nosso ver, um olhar mais detalhado, quais sejam: (i) há muitos outros adjuntos temporais que praticamente nunca foram analisados, como 'durante $\mathrm{X}$ tempo, 'de $\mathrm{X}$ a $\mathrm{Y}$

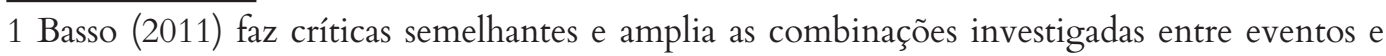
adjuntos. 
R. M. Basso \& J.

F. BERgamini-

Perez

A semântica de vetores: uma proposta de análise para os adjuntos temporais

tempo', etc., e (ii) a própria estrutura dos adjuntos foi pouco explorada, mas basta um rápido olhar para notarmos que eles são compostos, via de regra, de uma preposição mais um sintagma de medida temporal (i.e., 'X tempo').

Neste artigo, exploraremos essas três questões (a saber: outras possibilidades de combinação de adjunto temporais e eventos, uma maior diversidade de adjuntos temporais, e sua estrutura) através de uma teoria conhecida como "vector space semantics" (VSS). Tal teoria foi desenvolvida para lidar inicialmente com modificadores espaciais, mas dadas as relações íntimas entre a descrição espacial e temporal nas línguas naturais, propomos que podemos usar tal teoria com muito sucesso e economia para a investigação dos adjuntos temporais, principalmente no que diz respeito às questões específicas que colocamos aqui.

Para dar conta de nossos objetivos, o presente artigo está organizado da seguinte forma: na seção 1, introduziremos nossos dados, ou seja, os adjuntos que exploraremos, as combinações entre adjuntos e eventos, bem como notas sobre a estrutura composicional desses adjuntos; na seção 2, apresentaremos a VSS; e, finalmente, na seção 3, propomos uma análise de nossos dados nos moldes da VSS. Na conclusão, retomaremos o caminho percorrido, e notaremos os ganhos e os problemas em aberto.

\section{Eventos e adjuntos}

Nesta seção, teceremos algumas considerações sobre eventos e adjuntos temporais que guiaram nossas discussões, bem como sobre o tipo de teoria que procuramos desenvolver e sobre o domínio empírico que visamos explicar. Começaremos pelos eventos, passando depois para os adjuntos.

\subsection{Eventos}

Ao lidarmos com a análise semântica de eventos em língua natural, as categorias mais comumente analisadas são (a) a referência temporal, que lida com a questão sobre quando um dado evento ocorreu, e pode ser respondida de modo simplificando com "passado", "presente" e "futuro"2; (b) o aspecto verbal, que tem a ver com a representação do evento entre concluso (i.e., não mais sendo o caso num dado momento de consideração) e não-concluso (i.e., não necessariamente não mais sendo o caso num dado momento de consideração) ${ }^{3}$; e, finalmente, (c) a acionalidade do evento, que remete, em geral, às classes vendlerianas que nada mais são do que a organização de eventos em função de propriedades como sua

Revista Letras,

Curitiba, UFPR, n. 96 , pp.33-53, jul./dez. 2017. ISSN 2236-0999 (versão eletrônica)

2 O estudo da referência temporal sofreu uma enorme influência das ideias de Reichenbach (1949), que molda ainda hoje as principais abordagens sobre o assunto.

3 Encontramos aqui a conhecida oposição entre aspecto perfectivo (i.e., concluso) e aspecto imperfectivo (comumente subdividido entre habitual, progressivo e outros). 
duração, dinamicidade e a existência ou não de uma meta (um telos). A tabela abaixo traz as classes vendlerianas e seus traços mais comuns ${ }^{4}$ :

\begin{tabular}{|l|l|l|l|}
\hline & {$[$ durativo] } & [télico] & [dinâmico] \\
\hline accomplishments & + & + & + \\
\hline achievements & - & + & + \\
\hline Estativos & + & - & - \\
\hline Atividades & + & - & + \\
\hline
\end{tabular}

Tabela 1. Classificação vendleriana dos eventos

No presente artigo, não lidaremos com referência temporal e consideraremos todos os nossos exemplos, de modo confessadamente simplificado, como estando no passado. Também com relação ao aspecto, focaremos somente no aspecto perfectivo, entendido como sendo um modo de veicular um evento que não é mais o caso, que já cessou. Contudo, é importante deixarmos claro nosso entendimento da combinação perfectivo+télico no português brasileiro (PB).

Segundo Basso (2007; 2011) e Basso e Pires de Oliveira (2010), o perfectivo em $\mathrm{PB}$, quando combinado com eventos télicos, não garante o alcance do telos; dito de outra maneira, as sentenças abaixo não garantem que o quadro está pronto (1), o quarto está arrumado (2) e a casa está acabada (3), como indicam as continuações sugeridas:

(1) João pintou o quadro (mas somente terminará amanhã).

(2) João arrumou o quarto (mas não acabou).

(3) João construiu a casa (mas só até acabar o dinheiro da poupança).

Portanto, o perfectivo em PB pode ser considerado fraco ou neutro, no sentido de que ele apenas indica que um evento (télico ou não) não é mais o caso, sem garantir que o telos dos eventos télicos tenha sido alcançado.

Voltando à tabela acima, podemos notar como os adjuntos temporais são relevantes, dado que, idealmente, 'por X tempo' só combinaria com eventos durativos e 'em $\mathrm{X}$ tempo', com eventos télicos, como nos exemplos abaixo:

(4) João desenhou uma casa por 30 minutos. (acc. / traço + durativo)

(5) ?? João cruzou a linha de chegada por 5 minutos.

(6) João passeou por 20 minutos

4 Além das classes apresentadas na tabela 1, é possível pensar também, pelo menos, na classe dos semelfactivos, cujos traços seriam [- durativo] e [- télico], e também na classe dos degree achievements, eventos télicos graduais que em muitos testes se comportam como atividades. Não exploraremos essas classes neste artigo, mas argumentamos que nossas considerações, bem como as análises em VSS, podem ser facilmente estendidas a elas. 
R. M. Basso \& J.

F. BERgamini-

Perez

A semântica de vetores: uma proposta de análise para os adjuntos temporais

(7) João teve fome por 15 minutos. (est. / traço + durativo)

(8) João desenhou uma casa em 30 minutos. (acc. / traço + durativo)

(9) João cruzou a linha de chegada em 5 minutos.

(10) ?? João passeou em 20 minutos

(11) ?? João teve fome em 15 minutos. (est. / traço + durativo)

Com relação ao adjunto 'por X tempo', sua combinação com eventos atélicos durativos simplesmente veicula quanto tempo o dado evento durou, e o mesmo vale para accomplishment (télicos durativos), notando que, nesse caso, há uma forte indicação de que o telos não foi alcançado - é o que chamamos de interpretação de "detelicização". Para os achievements, que são não-durativos, a combinação não é possível, salvo em casos em que o evento pode ser interpretado como uma atividade, como no exemplo (12); nesse caso, a interpretação é que por 5 minutos João ficou atrás no placar, mas sem saber se ele de fato perdeu o jogo.

(12) João perdeu o jogo por 5 minutos.

Passando ao adjunto 'em X tempo', vemos que ele claramente indica que o telos de eventos télicos foi alcançado - uma interpretação que podemos chamar, portanto, de "télica". Para o caso de eventos atélicos, a única interpretação possível, provavelmente alcançada via coerção, é a "incoativa"; segundo tal interpretação, para uma sentença como (10) o que temos é que o adjunto mede quanto tempo João levou para começar a passear, e não quanto tempo ele ficou passeando. Suponha que depois de chegar em casa, João troque de roupa, escove os dentes, feche a casa e só então saia para passear; em tal cenário, podemos dizer:

(13) João passeou em 20 minutos,

Indicando assim o tempo que ele levou para passear.

Podemos resumir essa discussão nas duas tabelas abaixo ("t" e "d" se referem, respectivamente, aos traços de telicidade e duratividade):

\begin{tabular}{|l|l|l|l|}
\hline Perfectivo & t & d & interpretação \\
\hline 14. João arrumou o quarto por 10 minutos. & + & + & detelicização \\
\hline 15. João perdeu o jogo por 10 minutos. & + & - & detelicização \\
\hline 16. João nadou por 10 minutos. & - & + & duração do evento \\
\hline 17. João ficou triste por 10 minutos. & - & + & duração do evento \\
\hline
\end{tabular}

Tabela 2. Sentenças com a combinação do adjunto 'por $\mathbf{X}$ tempo'

Revista Letras,

Curitiba, UFPR, n. 96 , pp.33-53, jul./dez. 2017. ISSN 2236-0999 (versão eletrônica) 


\begin{tabular}{|l|l|l|l|}
\hline Perfectivo & t & d & interpretação \\
\hline 18. João arrumou o quarto em 10 minutos. & + & + & télica \\
\hline 19. João perdeu o jogo em 10 minutos. & + & - & télica \\
\hline 20. João nadou em 10 minutos. & - & + & incoativa \\
\hline 21. João ficou triste em10 minutos. & - & + & incoativa \\
\hline
\end{tabular}

Tabela 3. Sentenças com a combinação do adjunto 'em X tempo'

Como especificado acima, nosso intuito é capturar essas e outras combinações de adjuntos temporais e eventos no quadro da "semântica de vetores espaciais"; antes de apresentar essa teoria, no entanto, é importante fazermos algumas observações sobre a semântica e a estrutura dos adjuntos temporais, tópico da seção a seguir.

\subsection{Adjuntos}

Talvez uma das primeiras abordagens a lidar, nos termos atuais da semântica formal, com a interpretação dos adjuntos temporais seja Dowty (1979). Em seu trabalho, o autor afirma que adjuntos como 'for X time' e 'in X time' (equivalentes, mas não idênticos, a 'por X tempo' e 'em X tempo') podem ser entendidos como relacionando eventos e seu desenvolvimento a medidas de tempo, de modo que, grosso modo, 'for X time' relaciona soma de eventos de um mesmo tipo a (sub)intervalos temporais e 'in X time' relaciona eventos únicos (ou atômicos) a um único intervalo temporal. Essa intuição explica, em princípio, a compatibilidade de 'for X time' com eventos atélicos (que podem ser entendidos como somas mereológicas de eventos do mesmo tipo) e 'in X time' com télicos (que seriam atômicos, não possuindo partes próprias). Rothstein (2004) lança mão das mesmas intuições para lidar e caracterizar esses adjuntos no quadro da "semântica de eventos", como abaixo (adaptado de Rothstein (2004, p. 178)):

$$
\begin{aligned}
& {[[\text { in an hour }]]=\lambda \mathrm{P} \lambda \mathrm{e} \cdot \mathrm{P}(\mathrm{e}) \wedge \forall \mathrm{e}^{\prime}\left[\mathrm{e}^{\prime} \in \operatorname{ATOM}(\mathrm{P}) \rightarrow \mathrm{\tau}\left(\mathrm{e}^{\prime}\right) \subseteq 1 \text { HOUR }\right]} \\
& {[[\text { for an hour }]]=\lambda \mathrm{P} \cdot \exists \mathrm{e}\left[\mathrm{\tau}(\mathrm{e})=1 \mathrm{HOUR} \wedge \forall \mathrm{i} \sqsubseteq \mathrm{\tau}(\mathrm{e}) \exists \mathrm{e}^{\prime}\left[\mathrm{P}\left(\mathrm{e}^{\prime}\right) \wedge \subseteq \mathrm{e}^{\prime} 1 \mathrm{e} \wedge \mathrm{\tau}\left(\mathrm{e}^{\prime}\right)=\mathrm{i}\right]\right]}
\end{aligned}
$$

Contudo, conforme argumenta Basso (2011), com tal caracterização, as possiblidades de interpretação apresentadas nas tabelas 2 e 3 acima, quando a combinação não é télico + 'em X tempo' nem atélico + 'por X tempo', são perdidas; é, portanto, necessário pensar em outra estratégia para lidar com esses adjuntos.

Além disso, como notamos, não temos apenas esses dois adjuntos, e a intuição por trás de fórmulas como as discutidas acima dificilmente pode ser levada a outros adjuntos, o que é mais um motivo para pensarmos no quadro de uma teoria mais ampla (i.e., que dê conta de maneira orgânica das possibilidades 
R. M. BASSO \& J.

F. BERGAMINI-

Perez

A semântica de vetores: uma proposta de análise para os adjuntos temporais

de combinações encontradas) - conforme argumentaremos a VSS é justamente uma teoria desse tipo.

Antes de apresentarmos a VSS, é importante falarmos um pouco sobre a composição dos adjuntos temporais. Tais adjuntos têm basicamente duas partes: uma preposição seguida por uma construção de sintagma de medida (SM). Assim, temos, por exemplo:

'em 1 hora' = em X tempo = preposição + SM

'por 25 minutos' $=$ por $\mathrm{X}$ tempo $=$ preposição $+\mathrm{SM}$

O mesmo vale para adjuntos como 'durante $\mathrm{X}$ tempo' e 'até $\mathrm{X}$ tempo'; por sua vez, 'de $\mathrm{X}$ a $\mathrm{Y}$ tempo' tem uma estrutura ligeiramente diferente, mas ainda assim é composto por preposição e SM.

O sintagma de medida que se combina com uma dada preposição para resultar num adjunto temporal pode ser de natureza diferente, e, num primeiro olhar, podemos classificar os SMs como:

a) SMs primários: são cronológicos ou envolvem alguma medição explícita de tempo, como 'manhã', 'verão', '10 minutos', '1 hora', etc;

b) SMS secundários: são dados por elementos não cronológicos que podem ser tomados como referência e/ou parâmetro de duração, como 'jogo', 'filme', 'peça', etc.

c) SMs pontuais: não remetem a um momento de tempo, mas sim a um evento quer serve como delimitação de uma duração, como 'anoitecer', 'ouvir um barulho', etc.

Tomemos 'em X tempo' e 'até X tempo' como exemplos. O primeiro combina-se apenas com SMs primários, e o segundo, somente SMs com pontuais:

(22) João desenhou um círculo em 1 hora/ ? em o (no) jogo / ? em o (no) anoitecer.

(23) João correu ? até 1 hora / ? até o jogo / até anoitecer.

Revista Letras,

Curitiba, UFPR, n. 96, pp.33-53, jul./dez. 2017. ISSN 2236-0999 (versão eletrônica)

5 Algumas combinações em (21) são aceitáveis, mas não com um sentido temporal; em (22), 'até' pode ser interpretado como 'no máximo', mas não é essa a interpretação que buscamos e sim a de limite exato. 
Em resumo, temos uma combinatória nas seguintes linhas (tabela retirada de Basso e Bergamini-Perez, 2016, p. 366):

\begin{tabular}{|l|l|l|l|l|l|}
\hline SMs & \multicolumn{5}{|l|}{ Adjuntos } \\
& em X tempo & por X tempo & durante X tempo & de X a Y tempo & até X tempo \\
\hline Primário & OK & OK & OK & OK & $\varnothing$ \\
\hline Secundário & $\varnothing$ & $\varnothing$ & OK & OK & $\varnothing$ \\
\hline Pontual & $\varnothing$ & $\varnothing$ & $\varnothing$ & $\varnothing$ & OK \\
\hline
\end{tabular}

Tabela 4. Combinação dos sintagmas de medida com os diferentes tipos de adjuntos

São essas as principais noções do domínio da semântica de eventos de que precisamos e são esses os tipos de dados a serem explicados. Na seção seguinte, passaremos a uma exposição da VSS para então apresentar nossa proposta de análise.

\section{Vector space semantics (VSS)}

A “semântica de vetores espaciais" (ou vector space semantics, VSS) é uma teoria proposta inicialmente por Zwarts (1997), e depois ampliada por diversos autores, principalmente Zwarts e Winter (2000). Em linhas gerais, a VSS, conforme apresentada por Zwarts (1997) e Zwarts e Winter (2000), tem como base e como objetivo principal a investigação de relações espaciais, notadamente de modificadores espaciais, e reconhece como primitivo ontológico fundamental vetores $\mathrm{v}^{6}$, com valores determinados nos números reais $\mathrm{R}$.

O interessante da proposta feita pelos autores, principalmente considerando sua análise da interpretação das preposições, reside no fato de que as preposições estabelecem uma posição relativa para o referente do item lexical ao qual estão ligadas e, para, capturar as diferenças entre as diversas preposições, os autores

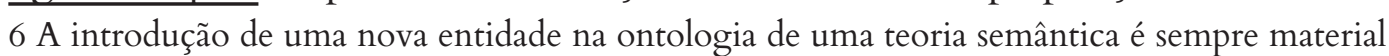
de controvérsia, pois pode comprometer a parcimônia ontológica da teoria e precisa de uma argumentação empírica e lógica consistente. Nos estudos linguísticos, isso aconteceu com a noção de "eventos", e vem acontecendo com as noções de "espécie" e "grau", cuja introdução na ontologia conta com um grande aparato empírico, baseado tanto em intuições quanto em descrições linguística, mas ainda encontra resistência por parte de autores que argumentam dar conta do problemas linguísticos e intuições sem uma nova entidade na ontologia. Os argumentos a favor da introdução de vetores na ontologia fogem um pouco aos objetivos do presente artigo, mas é importante mencionar, ainda que em linhas gerais, as motivações e vantagens de contarmos com essa nova entidade. A principal motivação para contarmos com vetores é resolver problemas composicionais de sintagmas preposicionais locativos combinados com sintagmas de medição - como apresentam Zwarts (1997) e Zwarts e Winter (2000), as abordagens tradicionais não resolvem de maneira satisfatória os problemas colocados por esse tipo de construção. Em momentos posteriores, a VSS, e a ideia de vetores, foi mobilizada para lidar com diversas construções linguísticas que envolvem medidas, como as estruturas comparativas e adjetivos graduais, como nos trabalhos de Faller (1998, 2000), e fenômenos do domínio verbal (cf. MORZYCKI, 2006). Os vetores da VSS também são usados para lidar com trajetórias, o formato e o tamanho de objetos e demais fenômenos que têm relação com a linguagem espacial (cf. ZWARTS, 2003). A análise que apresentamos aqui também pode ser vista como mais um argumento a favor de termos vetores na ontologia. Contudo, como não podia deixar de ser, esse tema ainda é controverso e precisa de maiores desenvolvimentos. Agradecemos a um dos pareceristas anônimos que nos chamou a atenção para esta, e outras, importantes questões. 
R. M. BASSO \& J.

F. Bergamini-

Perez

A semântica de vetores: uma proposta de análise para os adjuntos temporais

propõem a "Condição de Modificação", que nada mais é que considerar a preposição como um vetor que é compreendido como um segmento direcionado que aponta de uma localização espacial para outra. Para entendermos a ideia por trás dessa condição, podemos considerar, por exemplo, o caso de um avião estar voando sobre as nuvens e alguém pronunciar a sentença,

(24) O avião está sobre as nuvens.

De acordo com essa condição, temos que o avião se localiza em relação a um outro objeto (i.e. as nuvens), sendo dado então um objeto de referência (as nuvens) e um objeto localizado (o avião). Ou seja, para que essa condição de modificação exista e, consequentemente, a análise pelos vetores seja possível, é necessário que existam um objeto de referência e um objeto localizado, como pode ser exemplifica pela sentença (24); a preposição estabelece então uma relação entre eles.

Apresentamos, na sequência abaixo, como é o tratamento que a VSS oferece para PPs locativos, tomando como exemplo 'embaixo da prateleira':

(25a) $[$ embaixo da prateleira $]]=\lambda_{v}[\mathrm{v}$ começa na localização da prateleira $\wedge \mathrm{v}$ aponta para baixo]

(25b) [[o livro está embaixo da prateleira $]]=\exists \mathrm{v}$ [v começa na localização da prateleira ${ }^{\wedge} \mathrm{v}$ aponta para baixo ${ }^{\wedge} \mathrm{v}$ termina na localização do livro]

Como podemos ver, nessa teoria, os PPs locativos denotam conjuntos de vetores. Considerando um PP como "embaixo da prateleira", o SM "10 centímetros" indica o tamanho do vetor v:

(25c) $[[10$ centímetros $]]=\lambda \mathrm{v} \cdot \mathrm{v}$ mede 10 centímetros

(25d) $[[10$ centímetros embaixo da prateleira $]]=$ $\lambda \mathrm{v} .[[10$ centímetros $]](\mathrm{v}) \wedge[[$ embaixo da prateleira $]](\mathrm{v})=$ $\lambda_{\mathrm{v}}\left[\mathrm{v}\right.$ mede 10 centímetros $^{\wedge} \mathrm{v}$ começa na localização da prateleira $\wedge \mathrm{v}$ aponta para baixo]

(25e) [[o livro está 10 centímetros embaixo da prateleira $]]=\exists \mathrm{v}[\mathrm{v}$ mede 10 centímetros ${ }^{\wedge} \mathrm{v}$ começa na localização da prateleira ${ }^{\wedge} \mathrm{v}$ aponta para baixo ${ }^{\wedge} \mathrm{v}$ termina na localização do livro]

Em suas análises, a VSS considera uma série de propriedades matemáticas associadas aos vetores, sua decomposição e combinação, como as seguintes:

Revista Letras,

Curitiba, UfPR, n. 96, pp.33-53, jul./dez. 2017. ISSN 2236-0999 (versão eletrônica) 
a. O produto vetorial de dois vetores quaisquer ou de um vetor e um número real é feito a partir de um operador de multiplicação escalar dado por '., como por exemplo:

$$
\mathrm{V} \cdot \mathrm{W}
$$

b. Existe um operador de adição ‘+', que possibilita a soma vetorial.

$$
\begin{aligned}
& \rightarrow \quad \rightarrow \\
& \mathrm{V}+\mathrm{W}
\end{aligned}
$$

A figura abaixo ilustra as operações vetoriais descritas na sequência:

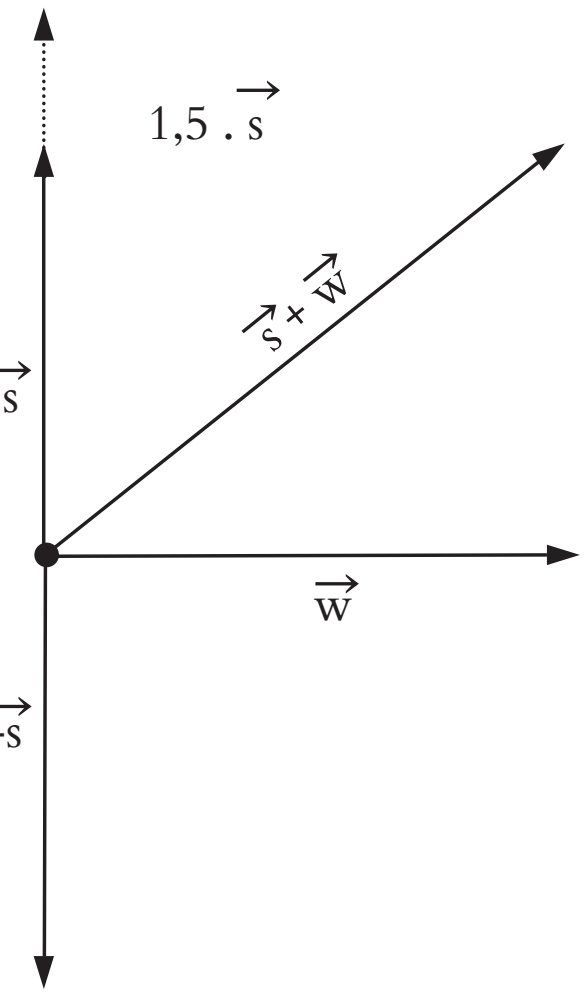

Figura 1. Vetores e operações vetoriais

i. Para todo $\mathbf{s}$ e $\mathbf{w} \in \mathbf{V}, \mathbf{s}+\mathbf{w}=\mathbf{w}+\mathbf{s}$

ii. Para todo $\mathbf{s}, \mathbf{w}$ e $\mathbf{v} \in \mathbf{V},(\mathbf{s}+\mathbf{w})+\mathbf{v}=\mathbf{s}+(\mathbf{w}+\mathbf{v})$

iii. Existe um elemento $0 \in \mathbf{V}$, o vetor zero, no qual $\mathbf{s}+0=0+\mathbf{s}=\mathbf{s}$ para todo $\mathbf{s} \in \mathbf{V}$

iv. Para todo $\mathbf{s} \in \mathbf{V}$ existe um $-\mathbf{s} \in \mathbf{V}$, o inverso de $\mathbf{s}$, no qual $\mathbf{s}+(-\mathbf{s})=\mathbf{0}$

v. Para todo $\mathbf{s}$ e $\mathbf{w} \in \mathbf{V}$ e $\mathbf{c} \in \mathbf{R}, \mathbf{c}(\mathbf{s}+\mathbf{w})=\mathbf{c s}+\mathbf{c w}$

vi. Para todo $\mathbf{s} \in \mathbf{V}$ e $\mathbf{c}$ e $\mathbf{d} \in \mathbf{R},(\mathbf{c}+\mathbf{d}) \mathbf{s}=\mathbf{c s}+\mathrm{ds}$ e $(\mathbf{c d}) \mathbf{s}=\mathbf{c}(\mathbf{d s})$

vii. Para todo $\mathbf{s} \in \mathbf{V}, 1 \mathbf{s}=\mathbf{s}$

Esse tratamento simples, econômico e robusto, que conta com uma elaborada linguagem formal, foi levado para outros domínios além do espacial, 
R. M. Basso \& J.

F. BERGAMINI-

Perez

A semântica de vetores: uma proposta de análise para os adjuntos temporais

como a semântica de adjetivos graduais e de medidas ("the semantics of measurement") (cf. FALLER, 2000)7:

(26) $[[10$ centímetros mais alto que o João]] =

$\lambda \mathrm{v} \cdot[[10$ centímetros $]](\mathrm{v}) \wedge[[$ mais alto que o João $]](\mathrm{v})=$ $\lambda \mathrm{v}$ [v mede 10 centímetros ${ }^{\wedge} \mathrm{v}$ começa na altura de João (sua localização na escala de altura) ^ $\mathrm{v}$ aponta para cima]

A extensão das ferramentas da VSS para o domínio temporal é um passo até certo ponto natural, dadas as similaridades entre a fala sobre espaço e a fala sobre o tempo. Como exemplo de uma das primeiras dessas extensões, Morzycki (2006), lançando mão de alguns conceitos da semântica de vetores, define perfectividade como abaixo ${ }^{8}$ :

(27) $[[$ Perfectivo $]]=\lambda \mathrm{f}_{<s, \mathrm{t}\rangle} \lambda \mathrm{v} \cdot \exists \mathrm{e}\left[\mathrm{f}(\mathrm{e})^{\wedge} \mathrm{\tau}(\mathrm{e}) \subseteq \mathrm{v}\right]$

Ou seja, um evento perfectivo $f$ é aquele cujo tempo de duração (i.e, running time, ou $\mathrm{t}(\mathrm{e}))$ está contido num vetor v; o tempo de duração do evento é menor ou igual ao vetor, configurando assim a perfectividade9. É importante notar também que essa definição de perfectividade é compatível com o que dissemos sobre o perfectivo e sua relação com telos no PB na seção 1.1.

O ponto chave, a nosso ver, é a associação da duração de um dado evento $(\tau(\mathrm{e}))$ a um vetor, que codificará justamente essa duração. Além disso, diferentemente do espaço, os vetores usados em nossa análise temporal terão somente uma direção e sentido, dado que não contaremos "tempo negativo". Passemos então à análise dos adjuntos temporais do PB utilizando a VSS.

\section{VSS e adjuntos temporais}

Nesta seção, analisaremos os seguintes adjuntos temporais do $\mathrm{PB}$, na seguinte ordem: 'por $\mathrm{X}$ tempo', 'em $\mathrm{X}$ tempo', 'durante $\mathrm{X}$ tempo', 'de $\mathrm{X}$ a $\mathrm{Y}$ tempo', e 'até $\mathrm{X}$ tempo'.

7 A ideia é que sintagmas de medida denotam "tamanhos" (i.e., medidas) de vetores, que podem pertencer a vários domínios - espaciais, temporal, dimensional, escalar (relacionados a adjetivos escalares).

8 A fórmula para o aspecto perfectivo utilizada por Morzycki (2006) é inspirada nas ideias Klein (1994).

9 Como nota Morzycki (2006), o que temos em (27) é a denotação do aspecto e não do evento ou do verbo, que continuam a possuir a mesma denotação tradicional. A intuição interessante é

Revista Letras, Curitiba, UFPR, n. 96 , pp.33-53, jul./dez. 2017. ISSN 2236-0999 (versão eletrônica) que aspecto tem a ver com o modo como o verbo se relaciona com (a medida de) seu tempo de desenvolvimento, e por isso o uso de vetores é interessante. 


\section{1. 'por $\mathbf{X}$ tempo'}

Tomemos as sentenças da tabela 2, acima, devidamente renumeradas:

\begin{tabular}{|l|l|l|l|}
\hline perfectivo & t & d & interpretação \\
\hline 28. João arrumou o quarto por 10 minutos. & + & + & detelicização \\
\hline 29. João perdeu o jogo por 10 minutos. & + & - & detelicização \\
\hline 30. João nadou por 10 minutos. & - & + & duração do evento \\
\hline 31. João ficou triste por 10 minutos. & - & + & duração do evento \\
\hline
\end{tabular}

Tabela 5. Sentenças com a combinação do adjunto 'por $\mathbf{X}$ tempo'

Uma proposta para 'por $\mathrm{X}$ tempo' é dizer que ele simplesmente atribui um valor, via seu SM, ao vetor associado à duração do evento. Consideremos as sentenças abaixo:

(28) João arrumou o quarto por 10 minutos.

(28a) João arrumou o quarto.

Em ambos os casos, sabemos que João arrumou o quarto, mas não sabemos se ele necessariamente terminou a arrumação (i.e., se o evento alcançou seu telos); mas, com (27), sabemos também por quanto tempo esse evento foi o caso.

Considerando, "AQ" como 'arrumar o quarto', chegamos às seguintes formulações ${ }^{10}$ :

$\left(28^{\prime}\right)[[$ João arrumou o quarto por 10 minutos $]]=\lambda v \cdot \exists e[A Q(e) \wedge A G(e, j)$ ${ }^{\wedge} \mathrm{T}(\mathrm{e}) \subseteq \mathrm{v}^{\wedge} \mathrm{v}=10$ minutos]

$\left(28 \mathrm{a}^{`}\right)[[$ João arrumou o quarto $]]=\lambda \mathrm{v} \cdot \exists \mathrm{e}\left[\mathrm{AQ}(\mathrm{e})^{\wedge} \mathrm{AG}(\mathrm{e}, \mathrm{j})^{\wedge} \mathrm{\tau}(\mathrm{e}) \subseteq \mathrm{v}\right]$

Em suma, a diferença entre a combinação "predicado + aspecto perfectivo" e a combinação "predicado + aspecto perfectivo + adjunto "por X tempo"” é somente que, na segunda, o adjunto atribui um valor do vetor v, que é dado pelo SM; nenhuma das sentenças, como dissemos, afirma que o telos de eventos télicos foi alcançado, mas somente que o evento não é mais o caso (i.e., está contido num vetor que captura o tempo), e uma delas (1) diz qual é o valor do vetor.

Com eventos télicos, o resultado é o que chamamos de detelicização, ou seja, não é possível determinamos se o evento teve seu fim (i.e., telos) alcançado ou não (cf. BASSO, 2007; 2011), e com eventos atélicos, o resultado é a medida da duração do evento, como no exemplo abaixo, em que "N" está por 'nadar':

10 Como notou um dos pareceristas anônimos, a análise dada para sentença em (28'), bem como as demais análises aqui apresentadas, trata as sentenças como denotando conjuntos de vetores, e não valores de verdade. Para chegar a tanto, é necessário fazer o "fechamento existencial" dos vetores, que é algo que consideramos ficar a cargo do contexto. Há ainda questões ligadas à derivação sintática desse tipo de estruturas que não trataremos aqui. Agradecemos ao parecerista por levantar essas importantes questões. 
R. M. Basso \& J.

F. BERGAMINI-

Perez

A semântica de vetores: uma proposta de análise para os adjuntos temporais $\left(30^{\prime}\right)[[$ João nadou por 10 minutos $]]=\lambda \mathrm{v} \cdot \exists \mathrm{e}[\mathrm{N}(\mathrm{e}) \wedge \mathrm{AG}(\mathrm{e}, \mathrm{j}) \wedge \mathrm{t}(\mathrm{e}) \subseteq \mathrm{v} \wedge$ $\mathrm{v}=10$ minutos $]$

Sendo assim, todo o resultado é composicional, e a detelicização é o resultado da combinação de eventos télicos com o adjunto 'por X tempo' que, em todos os casos, simplesmente atribui uma medida ao vetor associado à duração do evento.

Antes de passarmos ao adjunto 'em $\mathrm{X}$ tempo', salientamos que o resultado com estativos é o mesmo e com achievements há duas possibilidades: (i) o achievement é interpretado como uma atividade (como vimos com o exemplo (12), na seção 1.1), ou (ii) o SM do adjunto é grande o suficiente para permitir repetição, como em:

(32) João ganhou a corrida por 10 anos;

o exemplo (32) não trata de uma única vitória, mas sim de um número indefinido delas. Seja como for, em todos os casos, 'por X tempo' apenas fornece uma medida para o vetor:

(33) $[[$ por $\mathrm{X}$ tempo $]]=\lambda_{\mathrm{v}} \cdot \mathrm{v}$ mede $\mathrm{X}$ tempo

\section{2 'em X tempo'}

Retomemos a tabela 3, devidamente renumerada:

\begin{tabular}{|l|l|l|l|}
\hline Perfectivo & t & d & interpretação \\
\hline 34. João arrumou o quarto em 10 minutos. & + & + & télica \\
\hline 35. João perdeu o jogo em 10 minutos. & + & - & télica \\
\hline 36. João nadou em 10 minutos. & - & + & incoativa \\
\hline 37. João ficou triste em10 minutos. & - & + & incoativa \\
\hline
\end{tabular}

Tabela 6. Sentenças com a combinação do adjunto 'em X tempo'

As interpretações geradas ao combinarmos eventos com 'em $\mathrm{X}$ tempo' são bem diferentes daquelas que vimos para 'por X tempo'. Começando pelos eventos perfectivos télicos, vemos que sua combinação com 'em $\mathrm{X}$ tempo' gera uma interpretação na qual o telos do evento foi alcançado; vejamos:

(34a) João arrumou o quarto em 10 minutos, ?? mas não terminou.

(34b) João arrumou o quarto por 10 minutos, mas não terminou.
Revista Letras,

Curitiba, UFPR, n. 96, pp.33-53, jul./dez. 2017. ISSN 2236-0999 (versão eletrônica) 
(34c) João arrumou o quarto em 10 minutos, e saiu pra brincar $=>$ quarto arrumado.

(34d) João arrumou o quarto por 10 minutos, e saiu pra brincar $\neq>$ quarto arrumado.

Ou seja, o adjunto ‘em X tempo' garante a interpretação de que o telos de um evento télico perfectivo foi alcançado.

Para capturar essa ideia, vamos considerar que esse adjunto pressupõe que o telos dos eventos télicos esteja contido na duração do evento - em outras palavras, teremos um evento terminado, que é a situação na qual o telos de um evento télico está contido em sua duração, de modo que ao término evento, seu telos já foi alcançado. Propomos ainda representar o telos de eventos télicos como $\mathrm{T}(\mathrm{e})$; assim, a pressuposição associada a 'em $\mathrm{X}$ tempo' pode ser representada por $[\mathrm{T}(\mathrm{e}) \subseteq \mathrm{\tau}(\mathrm{e})]$, como na fórmula em (38), em que $\delta \mathrm{X}$. representa um conteúdo pressuposicional:

(38) $\left[[e m X\right.$ tempo] $]=\lambda v \cdot \delta\left[T(e) \subseteq \mathrm{t}(\mathrm{e})^{\wedge} \mathrm{v}=\mathrm{x}\right] . \mathrm{v}$ mede $\mathrm{X}$ tempo

A diferença entre 'por X tempo' e 'em X tempo' se dá justamente por conta dessa pressuposição.

A análise de (34) fica como abaixo ${ }^{11}$ :

(34') [[João arrumou o quarto em 10 minutos $]]=\lambda v \cdot \exists e \cdot \delta[\mathrm{T}(\mathrm{e}) \subseteq \tau(\mathrm{e})]$. $\left[\mathrm{AQ}(\mathrm{e})^{\wedge} \mathrm{\tau}(\mathrm{e}) \subseteq \mathrm{v}^{\wedge} \mathrm{v}=10\right]$

Passando aos eventos atélicos, o que temos é uma leitura incoativa ${ }^{12}$, na qual, conforme vimos na seção 1.1, o adjunto não mede a duração do evento reportado na sentença principal, mas sim quanto tempo transcorreu para que esse evento tivesse início.

Uma maneira de interpretar a leitura incoativa é considerar que o adjunto 'em X tempo' mede agora uma quantidade de evento $\mathrm{N}$ cujo fim coincide com o início do evento reportado pela sentença principal. Essa intuição pode ser expressa como:

11 Omitiremos a representação do papel temático de agente para João para efeitos de simplificação.

12 É possível também que os eventos atélicos sejam interpretados como télicos, e assim "correr em 10 minutos" passa a ser "correr uma distância específica em 10 minutos"; se esse for o caso, cabe a análise proposta para (34'). 
R. M. Basso \& J.

F. BERGAMINI-

Perez

A semântica de vetores: uma proposta de análise para os adjuntos temporais
(39) $\left[[\right.$ perfect. + em X tempo] $]=\lambda_{v} \lambda f . \exists e \exists e ' . \delta\left[T\left(e^{\prime}\right) \subseteq \mathrm{\tau}\left(\mathrm{e}^{\prime}\right)\right] .\left[\mathrm{N}\left(\mathrm{e}^{\prime}\right)^{\wedge} \mathrm{f}(\mathrm{e})\right.$ $\left.{ }^{\wedge} \mathrm{T}\left(\mathrm{e}^{\ominus}\right) \subseteq \mathrm{v}^{\wedge} \mathrm{v}=\mathrm{X}^{\wedge} \mathrm{v}>\mathrm{i}(\mathrm{t}(\mathrm{e}))\right]$

em que "N" está por um ou mais eventos e' e " $v>i(\tau(e))$ " indica que o fim da medição do vetor coincide com o início da duração do evento e, que é o evento reportado pela sentença. Tomemos o exemplo (39), que "C" é 'correr':

(40) $[$ [joão correu em 10 minutos] $]=\lambda_{v} \exists \mathrm{e} \exists \mathrm{e} \cdot \delta\left[\mathrm{T}\left(\mathrm{e}^{\prime}\right) \subseteq \mathrm{t}\left(\mathrm{e}^{\prime}\right)\right] .\left[\mathrm{N}\left(\mathrm{e}^{\prime}\right)^{\wedge}\right.$ $\mathrm{C}(\mathrm{e})^{\wedge} \mathrm{\tau}\left(\mathrm{e}^{\prime}\right) \subseteq \mathrm{v}^{\wedge} \mathrm{v}=10$ minutos $\left.^{\wedge} \mathrm{v}>\mathrm{i}(\mathrm{t}(\mathrm{e}))\right]$

Em prosa: o conjunto eventos $\mathrm{N}$ está contido num vetor com duração de 10 minutos e o início do evento de correr coincide com o fim do vetor.

Essa análise captura os fatos de que, na leitura incoativa de (40), não sabemos por quanto tempo João correu nem quantos eventos levaram ao início da corrida. Além disso, ela preserva a intuição básica de que 'em X tempo' garante que o telos de eventos télicos está alcançado, ainda que ele seja o início do evento reportado na sentença.

Como é possível notar, as fórmulas em (38) e (39) são diferentes; atribuímos essa diferença a uma coerção, cujo correlato psicolinguístico foi detectado experimentalmente em Basso $(2007 ; 2008)$. A proposta é que tal coerção exige para o adjunto 'em X tempo' a estrutura apresentada em (39), que conta, entre outras coisas, com mais eventos do que simplesmente aquele reportado na sentença em que o adjunto aparece.

'por X tempo' e 'em X tempo' são os adjuntos mais estudados na literatura e eles encontram uma explicação interessante e orgânica também na VSS; vejamos agora outros adjuntos que podem ser analisados nos mesmos moldes. Comecemos por 'durante X tempo'.

\section{3 'durante X tempo'}

'durante X tempo' é muito semelhante a 'por X tempo', como demonstram as sentenças da tabela abaixo e suas interpretações:

\begin{tabular}{|l|l|l|l|}
\hline Perfectivo & t & d & interpretação \\
\hline 41. João arrumou o quarto durante 10 minutos. & + & + & detelicização \\
\hline 42. João perdeu o jogo durante 10 minutos. & + & - & detelicização \\
\hline 43. João nadou durante 10 minutos. & - & + & duração do evento \\
\hline 44. João ficou triste durante 10 minutos. & - & + & duração do evento \\
\hline
\end{tabular}

Tabela 7. Sentenças com a combinação do adjunto 'durante X tempo'

De fato, podemos entender 'durante $\mathrm{X}$ tempo' na VSS da mesma forma que 'por X tempo', com a diferença de que 'durante X tempo' aceita SMs primários e secundários:
Revista Letras,

Curitiba, UfPr, n. 96, pp.33-53, jul./dez. 2017. ISSN 2236-0999 (versão eletrônica) 
(41a) João arrumou o quarto durante 10 minutos / por 10 minutos.

(41b) João arrumou o quarto durante a partida de futebol / ?pela partida de futebol.

(43a) João nadou durante 10 minutos / por 10 minutos.

(43b) João nadou durante a partida de futebol / ?pela partida de futebol.

Essas sentenças podem ser analisadas como abaixo:

(41a') [[João arrumou o quarto durante 10 minutos $]]=\lambda v \cdot \exists e[A Q(e) \wedge \mathrm{T}(\mathrm{e})$ $\subseteq \mathrm{v}^{\wedge} \mathrm{v}=10$ minutos]

(41b') [[João arrumou o quarto durante a partida de futebol] $]=\lambda v \cdot \exists \mathrm{e}$ $\left[\mathrm{AQ}(\mathrm{e})^{\wedge} \mathrm{\tau}(\mathrm{e}) \subseteq \mathrm{v}^{\wedge} \mathrm{v}=\right.$ duração da partida de futebol $\left.{ }^{13}\right]$

(43a') [[João nadou durante 10 minutos] $]=\lambda \mathrm{v} \cdot \exists \mathrm{e}\left[\mathrm{N}(\mathrm{e})^{\wedge} \mathrm{T}(\mathrm{e}) \subseteq \mathrm{v}^{\wedge} \mathrm{v}=10\right.$ minutos]

(43b') [[João nadou durante a partida de futebol] $]=\lambda \mathrm{v} \cdot \exists \mathrm{e}\left[\mathrm{N}(\mathrm{e})^{\wedge} \mathrm{\tau}(\mathrm{e}) \subseteq \mathrm{v}\right.$ ${ }^{\wedge} \mathrm{v}=$ duração da partida de futebol]

Em suma, 'durante $\mathrm{X}$ tempo' fornece a medida temporal do vetor associado à duração dos eventos, que pode ser uma medida propriamente dita (i.e., SMs primários) ou um referencial externo de medida (i.e., SMs secundários), resultando nas mesmas interpretações apresentadas por 'por X tempo'.

\section{4 'de X a Y tempo'}

$\mathrm{O}$ adjunto 'de $\mathrm{X}$ a $\mathrm{Y}$ tempo' tem interpretações semelhantes aos adjuntos 'por X tempo' e 'durante $\mathrm{X}$ tempo', mas esses adjuntos se diferem na maneira em como seu SM é mobilizado. Consideremos as sentenças abaixo:

\begin{tabular}{|l|l|l|l|}
\hline Perfectivo & t & d & interpretação \\
\hline 45. João arrumou o quarto de 5 a 10 minutos. & + & + & detelicização \\
\hline 46. João perdeu o jogo de 5 a 10 minutos. & + & - & detelicização \\
\hline 47. João nadou de 5 a 10 minutos. & - & + & duração do evento \\
\hline 48. João ficou triste de 5 a 10 minutos. & - & + & duração do evento \\
\hline
\end{tabular}

Tabela 8. Sentenças com a combinação do adjunto 'de X à Y tempo'

É interessante notar como 'de X a Y tempo' se liga ao seu SM: o SM do adjunto fornecerá um valor para o vetor v que contém o evento que se situará entre X e Y. Consideremos a seguinte comparação:

13 Para tornar a fórmula mais transparente, poderíamos pensar numa função próxima à função " $\tau$ ", que tenha como argumento SMs secundários e como resultado durações; seja como for, essa decisão específica não interfere nos contornos de nossa análise. 
R. M. Basso \& J.

F. BERGAMINI-

Perez

A semântica de vetores: uma proposta de análise para os adjuntos temporais

(45’) [[João arrumou o quarto de 5 a 10 minutos $]]=\lambda v \cdot \exists \mathrm{e}[\mathrm{AQ}(\mathrm{e}) \wedge \mathrm{AG}(\mathrm{e}$, j) $\wedge \tau(\mathrm{e}) \subseteq \mathrm{v} \wedge \mathrm{z} \wedge 5 \mathrm{~min} \leq \mathrm{z} \leq 10 \mathrm{~min}]$

$\left(28^{\prime}\right)[[$ João arrumou o quarto por 10 minutos $]]=\lambda v \cdot \exists e[A Q(e) \wedge A G(e, j)$ $\wedge \tau(\mathrm{e}) \subseteq \mathrm{v} \wedge \mathrm{v}=10$ minutos]

(47’) [[João nadou de 5 a 10 minutos $]]=\lambda \mathrm{v} \cdot \exists \mathrm{e}[\mathrm{N}(\mathrm{e}) \wedge \mathrm{\tau}(\mathrm{e}) \subseteq \mathrm{v} \wedge \mathrm{v}=\mathrm{z} \wedge$ $5 \min \leq \mathrm{z} \leq 10 \mathrm{~min}]$

$\left(30^{\prime}\right)[[$ João nadou por 10 minutos $]]=\lambda \mathrm{v} \cdot \exists \mathrm{e}[\mathrm{N}(\mathrm{e}) \wedge \mathrm{\tau}(\mathrm{e}) \subseteq \mathrm{v} \wedge \mathrm{v}=10$ minutos]

$\mathrm{O}$ adjunto 'de $\mathrm{X}$ a $\mathrm{Y}$ tempo' admite, como vimos com os exemplos, não somente SMs primários, mas também secundários se eles se referem a dois pontos diferentes, de modo a um deles ser o início (i.e., 'de X') e um deles ser o fim (i.e., ‘a Y’) da medição, como em (49)-(51) (cf. BASSO; BERGAMINI-PEREZ, 2016).

(49) João estava correndo do início do jogo ao fim. (50) João arrumou o quarto da novela ao filme.

(51) João ficou triste do supermercado à sua casa.

Ainda é interessante notar, numa dada interpretação, 'de X a Y tempo' não toma o início do evento para realizar a medição de seu SM; antes, ele pode tomar um ponto qualquer no meio do desenrolar do evento em questão para ser o início de seu SM, estabelecendo uma mediação no desenrolar ou durante tal evento, independentemente de quando tal evento tenha começado, como no seguinte exemplo:

(52) João correu das 13 hs às $13: 35$ hs,

em que não necessariamente consideramos que o evento de correr do João tenha tido início às 13 hs. Seja como for, nossa análise captura essa interpretação, e a decisão entre essa e a interpretação em que X coincide com o início do evento fica a cargo da pragmática.

Passemos ao último adjunto temporal que consideraremos neste artigo.

\section{5 'até X tempo'}

Como nos últimos casos acima, o adjunto que 'até $\mathrm{X}$ tempo' é muito próximo a 'por X tempo':

\begin{tabular}{|l|l|l|l|}
\hline Perfectivo & t & d & interpretação \\
\hline 53. João arrumou o quarto até anoitecer. & + & + & detelicização \\
\hline 54. João perdeu o jogo até anoitecer. & + & - & detelicização \\
\hline 55. João nadou até anoitecer. & - & + & duração do evento \\
\hline 56. João ficou triste até anoitecer. & - & + & duração do evento \\
\hline
\end{tabular}

Tabela 9. Sentenças com a combinação do adjunto 'até $\mathbf{X}$ tempo'

Revista Letras,

Curitiba, UFPR, n. 96 , pp.33-53, jul./dez. 2017. ISSN 2236-0999 (versão eletrônica) 
Antes de apresentar nossa análise para 'até X tempo', é necessário fazermos duas observações. A primeira delas tem a ver com o SM de 'até $\mathrm{X}$ tempo', que, como vimos na seção 1.2, é pontual, pois não se refere a uma medida de tempo explícita (como os SMs primários) nem a eventos que fornecem uma duração (como os SMs secundários); esse adjunto tem como SM um outro evento que servirá como limite de sua medição:

(53) João arrumou o quarto até anoitecer / machucar o joelho.

(53a) ? João arrumou o quarto até 10 minutos.

(53b) ? João arrumou o quarto até a partida de futebo ${ }^{14}$.

A segunda observação tem a ver com a possibilidade de uma interpretação quantificacional de 'até' como algo nas linhas de ‘no máximo'. Nessa interpretação, a preposição 'até' pode aparecer no SM de quaisquer outros adjuntos:

(57) João estava desenhando uma casa em até 10 minutos.

(58) João estava desenhando uma casa por até 10 minutos.

(59) João estava desenhando uma casa durante até 10 minutos.

(60) João estava desenhando uma casa de 5 a até 10 minutos.

Os SMs das sentenças em (57)-(60) podem ser lidos como 'em no máximo 10 minutos', 'por no máximo 10 minutos', ‘durante no máximo 10 minutos’, ‘de 5 a no máximo 10 minutos'. Neste trabalho, não lidaremos com essa leitura, que podemos chamar de "quantificacional" de 'até', em que a preposição é interpretada no interior de um SM, mas somente com leituras como as da tabela 9, em que 'até' encabeça do adjunto temporal.

Feitas essas observações, propomos a seguinte análise para o adjunto 'até $\mathrm{X}$ tempo', em que “i( $(\mathrm{t}(\mathrm{e}))$ ” representa o início da duração do evento $e$ :

(61) $\left[\left[\right.\right.$ até $\mathrm{X}$ tempo]] $=\lambda \mathrm{P}_{\langle\mathrm{s}, \mathrm{t}\rangle} \lambda \mathrm{v} \cdot \exists \mathrm{e}[\mathrm{P}(\mathrm{e}) \wedge \mathrm{v}$ mede $\mathrm{z}$ tempo $\wedge \mathrm{z} \leq \mathrm{i}(\mathrm{\tau}(\mathrm{e}))]$

Consideremos agora (53):

(53’) $[[$ João arrumou o quarto até anoitecer $]]=\lambda \mathrm{v} \cdot \exists \mathrm{e}^{\prime} \exists \mathrm{e}\left[\mathrm{AN}\left(\mathrm{e}^{\prime}\right) \wedge \mathrm{AQ}(\mathrm{e})\right.$ $\left.\wedge \mathrm{t}(\mathrm{e}) \subseteq \mathrm{v} \wedge \mathrm{v}=\mathrm{z} \wedge \mathrm{z} \leq \mathrm{i}\left(\mathrm{\tau}\left(\mathrm{e}^{\prime}\right)\right)\right]$

A diferença mais fundamental entre 'até $\mathrm{X}$ tempo' e 'por $\mathrm{X}$ tempo' tem a ver com os SMs selecionados por um ou outro adjunto; argumentamos que 'até $\mathrm{X}$ tempo', como explicitado em (61), seleciona como seu SM um outro evento que servirá de limite temporal e dará a medida do vetor associado à duração do evento reportado.

14 A sentença (53b) é aceitável se considerarmos algo como 'até começar a partida de futebol', transformando o SM secundário em pontual. 
R. M. BASSO \& J.

F. Bergamini-

Perez

A semântica de vetores: uma proposta de análise para os adjuntos temporais

Revista Letras,

Curitiba, UFPR, n. 96, pp.33-53, jul./dez. 2017. ISSN 2236-0999 (versão eletrônica)

\section{Conclusão}

Neste artigo, nosso objetivo foi ampliar a análise dos adjuntos temporais comumente usados na semântica de eventos, aumentando a quantidade de combinações em que os adjuntos participam e mesmo a quantidade de adjuntos a serem investigados. Além disso, propomos que esses adjuntos devem ser entendidos por uma teoria mais robusta, que dê conta de suas interpretações num quadro teórico mais abrangente.

Diante de tal exigência, apresentamos e lançamos mão da chamada "vector space semantics" (VSS), uma teoria pensada primeiramente para lidar com modificadores espaciais, que tem vetores e suas operações como primitivos teóricos. Essa teoria já foi expandida para lidar com outros domínios e sua expansão para o domínio temporal é natural e econômica.

O resultado final a que chegamos para as intepretações de diversos adjuntos temporais e da perfectividade no português brasileiro é um quadro sucinto e coeso:

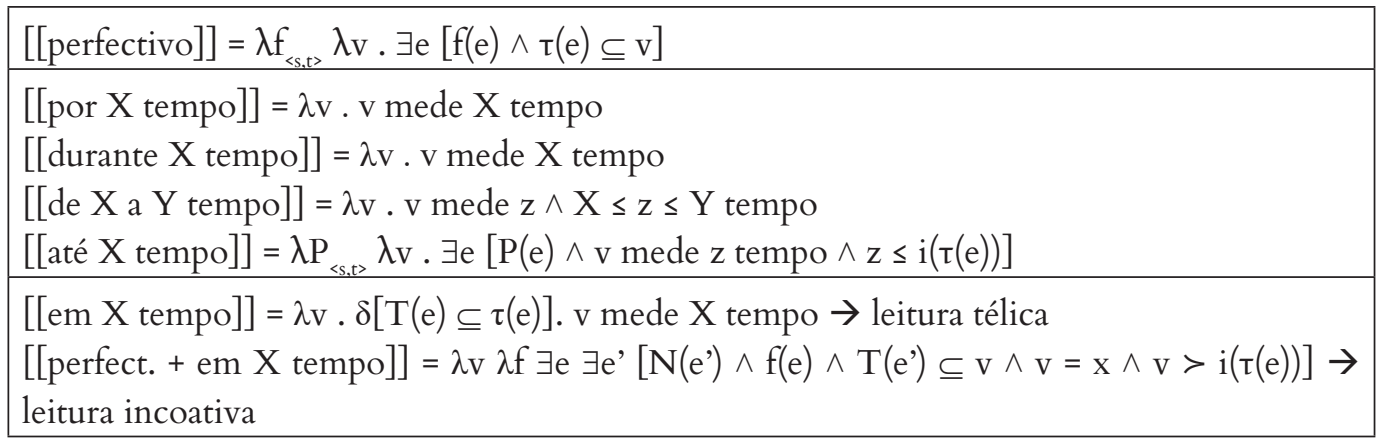

Tabela 10. Equações lógicas do aspecto perfectivo e de sua combinação com os adjuntos

Entre as diversas conclusões mostradas na discussão deste artigo, devemos ressaltar as duas possiblidades de interpretação que o adjunto 'em $\mathrm{X}$ tempo' possibilita, interpretação télica e interpretação incoativa, sendo importante salientar que a diferença desse adjunto em comparação com os outros adjuntos discutidos neste trabalho é o fato de ele lidar com uma função que chamamos de função telos $(\mathrm{T}(\mathrm{e}))$ e não com a função tempo-corrido.

Mesmo que tenhamos somente considerado o aspecto perfectivo, notamos que os adjuntos têm funções que vão além daquelas esperadas e tratadas por outros autores, principalmente pelo fato de poderem lidar com funções diferentes, dependendo do adjunto, como também com SMs distintos para cada caso, fazendo com que estes variem suas possibilidades de interpretação. Esse resultado aponta para a necessidade de também se entender o que ocorreria com a combinação destes adjuntos com eventos no aspecto imperfectivo.

Ressaltamos que a Semântica de Vetores (VSS) se revelou uma ferramenta eficaz na delimitação das funções de cada adjunto nas possíveis combinações examinadas, o que nos leva a crer que pode ser também uma ótima ferramenta semântica para a análise de outras singularidades investigadas pela semântica de eventos. 


\section{Referências}

BASSO, R. M. Telicidade e detelicização: semântica e pragmática do domínio tempo-aspectual.Dissertação(Mestrado em Linguística).Campinas: UNICAMP, 2007.

Coerção e detelicização: a psicolingüística e os fenômenos tempo-aspectuais. ReVEL. v. 6, n. 11, ago. 2008. ISSN 1678-8931.

. Uma proposta para Semântica dos adjuntos 'em X tempo' e 'por X tempo', Alfa, v. 55, n. 1, p.113-134, 2011.

BASSO, R. M.; BERGAMINI-PEREZ, J. F. O adjunto 'durante x tempo' no domínio tempo-aspectual. Letras de Hoje, v. 51, n. 3, p.367-374, 2016.

BASSO, R. M.; PIRES DE OLIVEIRA, R. 'em X tempo' e 'por X tempo' no domínio tempo-aspectual. Revista Letras, v. 81, p. 77-97, 2010.

DOWTY, D. Word Meaning and Montague Grammar. Dordrecht: Kluwer Academic Publishers, 1979.

FALLER, M. A vector space semantics for dimensional adjectives. ESSLLI 1998, 1998.

FALLER, M. Dimensional adjectives and measure phrases in vector space semantics. In FALLER, M.; KAUFMANN, S.; PAULY, M. (Eds.). Formalizing the Dynamics of Information. Stanford: CSLI Publications, 2000. p. 151-170.

KENNY, A. Action, Emotion and Will. London: Routledge, 1963.

KLEIN, W. Time in Language. London: Routledge, 1994.

MORZYCKI, M. Atelicity and Measure Phrases: Licensing Measure Phrase Modification Across AP, PP and VP. In: BAUMER, D.; MONTERO, D.; SCANLON, M. (Eds.) Proceedings of the $25^{\text {th }}$ West Coast Conference on Formal Linguistics. Somerville, MA: Cascadilla Proceedings Project, 2006. p. 279-287.

REICHENBACH, H. Elements of Symbolic Logic. Londres: MacMillan, 1947.

ROTHSTEIN, S. Events and Grammar. Dordrecht: Kluwer Academic Publishers, 1998.

. Structuring Events: A Study in the Semantics of Lexical Aspect. Malden: Blackwell Publishing Ltd, 2004.

VENDLER, Z. Verbs and Times. Philosophical Review, v. 66, n. 2, p. 143-160, 1957.

ZWARTS, J. Vector as relative positions: a compositional semantics of modified 
R. M. BASSO \& J.

F. Bergamini-

Perez

A semântica de vetores: uma proposta de análise para os adjuntos temporais

PPs. Journal of Semantics, v. 14, p. 57-86, 1997.

Vectors across spatial domains: From place to size, orientation, shape and parts. In: VAN DER ZEE, E.; SLACK, J. (Eds.). Axes and Vectors in Language and Space. Oxford: Oxford University Press, 2003. p. 39-68.

ZWARTS, J.; WINTER, Y. Vector space semantics: a model-theoretic analysis of locative prepositions. Journal of Logic, Language and Information, v. 9, p. 169$211,2000$.

Submetido: 21/02/217

Aceito: 30/06/2017

Revista Letras, Curitiba, UFPR, n. 96, pp.33-53, jul./dez. 2017. ISSN 2236-0999 (versão eletrônica) 